\title{
Antibacterial activity of Jatropha tanjorensis leaf extracts against bacteria associated with wound infections from the clinical setting
}

\author{
OLAWALE OLADEJO BABAYEMI ${ }^{1, \boldsymbol{v}}$, EBENEZER AYODELE OKE ${ }^{1,2}$, MICHAEL TOSIN BAYODE ${ }^{1}$ \\ ${ }^{1}$ Department of Microbiology, Federal University of Technology Akure. P.M.B 704, Akure, Ondo State 340110, Nigeria. Tel: +234-904-2422526, \\ "email: booladejo@futa.edu.ng \\ ${ }^{2}$ Department of Microbiology, Federal University Oye Ekiti. P.M.B 373, Oye Ekiti, Ekiti State 371101, Nigeria
}

Manuscript received: 13 October 2021. Revision accepted: 29 November 2021.

\begin{abstract}
Babayemi OO, Oke EA, Bayode MT. 2021. Antibacterial activity of Jatropha tanjorensis leaf extracts against bacteria associated with wound infections from the clinical setting. Nusantara Bioscience 13: 239-246. The use of microbial agents to treat infectious wounds based on ethnobotanical knowledge is still minimal. Antibacterial activity of Jatropha tanjorensis J.L.Ellis \& Saroja leaf crude extracts and commercial antibiotics were evaluated against bacterial isolates associated with wound samples using agar well diffusion and disc diffusion techniques, respectively. Phytochemical analysis of the sections was carried out using standard methods. Saponin $(58 \mathrm{mg} / \mathrm{g})$ was the highest phytochemical in the methanol extract, while flavonoid $(0.1 \mathrm{mg} / \mathrm{g})$ was the lowest percentage in the cold water extract. Methanol extract had the highest Zone of Inhibition (ZOI) of $33 \mathrm{~mm}$ against Staphylococcus aureus coagulasepositive. In contrast, the lowest ZOI $(5 \mathrm{~mm})$ against $S$. aureus coagulase-positive was obtained from cold water extract. Methanol extract resulted in the highest ZOI ranging from $16 \mathrm{~mm}$ to $17 \mathrm{~mm}$ against Escherichia coli, while the lowest ZOI was obtained from coldwater extract (3-4 mm). The highest Minimum Inhibitory Concentration (MIC) value of all extracts (100 $\mathrm{mg} / \mathrm{mL})$ was obtained against $P$. aeruginosa, while the lowest MIC value was obtained against coagulase-positive $S$. aureus $(12.5 \mathrm{mg} / \mathrm{mL})$. All extracts contain Octadecanoic acid, $n$-hexadecanoic acid, and phytol. This study revealed that methanol extract had the highest inhibitory activity against bacteria isolated from wound samples compared to other crude extracts and ciprofloxacin. Therefore, Jatropha tanjorensis could be used as a potent herbal remedy to reduce the adverse effects of wound infection.
\end{abstract}

Keywords: Antibacterial, bioactive compounds, extraction, Jatropha tanjorensis, phytochemical, wound

\section{INTRODUCTION}

A wound may be defined as a disruption in the epithelial integrity of the skin or as a loss or breaking of cellular and anatomic or functional continuity of living tissue (Murti et al. 2011). According to Wong et al. (2013), the human skin is a remarkably plastic organ that sustains insults and injury throughout life. The skin is under constant stress from the sun, smog, friction, tension, temperature, and other external factors. Therefore, under sufficient stress that causes injury, it results in wounds. Moreover, wounds may be classified as; open and closed, acute and chronic, avulsion and degloving, clean and contaminated, infected and colonized, laceration, incision and abrasion, puncture, penetration, and gunshot wounds (Escandon et al. 2011). Nonetheless, they exist in various forms comprising crush injuries, ulcers, skin tears, bruises, and post-operative, which directly or indirectly affect human health conditions. If it is not treated correctly, it may ultimately lead to death (Escandon et al. 2011). Various microorganisms, such as bacteria, fungi, parasites, and viruses, can cause wounds. Some commonly associated organisms include Staphylococcus aureus, Pseudomonas aeruginosa, Escherichia coli, Klebsiella spp., Acinetobacter spp., Candida albicans, and Bacteroides fragilis. The wound is one of the most common hospitalacquired infections, resulting in sepsis, limb loss, length of hospital stay, and higher costs (Shreshta and Sharma 2013).
Wound treatment is expected to alleviate any associated detrimental effects, as previously mentioned. Several factors affect wound healing, such as; aging, nutrition, obesity, repetitive trauma, skin moisture, chronic conditions, and medications (Guo and Di-Pietro 2010). Antibiotics have been of great value in treatment and prophylaxis for wound infections; however, several factors also affect reducing wound infections, i.e., the timing of administration, choice of antimicrobial agent, and durations of administration (Tatah et al. 2014). In addition, advances in infection control have not completely eradicated the wound infection problem due to drug resistance development (Mohammed et al. 2017).

Several species of plants have a significant therapeutic effect on alleviating the ailments of humankind (Yamac and Bilgili 2006). The genus Jatropha belongs to the family Euphorbiaceae. $J$. tanjorensis has been a potent ethnobotanical remedy in wound infection management (Ebana et al. 2019). Jatropha tanjorensis J.L. Ellis \& Saroja is a perennial herb distributed in rainforest zones of West Africa, including Nigeria (Iwalewa and Agbani 2005). It has several local names, i.e., Chaya leaf (English), Ugo-Oyibo (Igbo)", and is commonly referred to as "Hospital too far" in Pidgin English; Catholic vegetable, and "Iyana-ipaja" (Yoruba). They are predominant in the tropics and subtropics (Swarbrick 1997). This plant is mainly used for fencing but possesses many ethnobotanical 
and medicinal benefits in most parts of Southern Nigeria. The juice of Chaya leaf is used as herbal medicine.

Wounds result in the loss of epithelial continuity, and infections pose a significant health problem for patients. Most infectious microorganisms that cause wounds have developed resistance to most commercially available antibiotics. That has necessitated the search for novel sources of anti-infective compounds derived from medicinal plants to inhibit the growth of infectious bacteria (Ebana et al. 2019). This study evaluated the antibacterial activity of $J$. tanjorensis leaf extracts against bacteria associated with wound samples from the University Teaching Hospital, Ado-Ekiti, Nigeria.

\section{MATERIALS AND METHODS}

\section{Collection of Jatropha tanjorensis leaves}

Fresh leaves of $J$. tanjorensis were collected from a local market in Ado-Ekiti, Ekiti State, South-West, Nigeria. The plant was identified and authenticated by a plant scientist in the Department of Crop, Soil, and Pest Management, Federal University of Technology, Akure. The leaves were rinsed using clean water and air-dried for three weeks to a constant weight and then pulverized using an electric blender (Binatone blender/grinder-BLG 450). The pulverized plant material was kept in an air-tight plastic container for further use.

\section{Collection of wound swabs}

A total of 259 wound swabs were collected from various types of wound samples ranging from burns, chronic arterial foot ulcers, Diabetes Mellitus foot ulcers, post skin graft wound dehiscence, chronic leg ulcer, and avulsion wound at the wound care unit of the Ekiti State University Teaching Hospital, Ado-Ekiti, Nigeria. The wounds were cleaned with a cotton swab moistened with normal saline. The swab was aseptically obtained using the Levine wound collection method (Levine et al. 1976) by rotating the tip of the cotton swab over at least a $1 \mathrm{~cm}^{2}$ area of viable wound bed tissue for 5 seconds with sufficient pressure to extract fluid from the wound tissue of each patient without contamination. The wound swab samples were transported to the Microbiology Laboratory of the Federal University Oye Ekiti, Nigeria, for bacteriological analysis.

\section{Isolation of bacteria from wound swabs}

The collected swab sticks of wound samples were inoculated on a nutrient agar slant and incubated at $37^{\circ} \mathrm{C}$ for 24 to 48 hours (Mulu et al. 2013). Colonies formed after incubation were sub-cultured on blood agar, mannitol salt agar (MSA) (Hi-Media, India), and eosin methylene blue (EMB) agar (Hi-Media, India) to produce pure, distinct colonies. These were further subjected to various biochemical tests for bacterial identification concerning Bergey's manual of determinative Bacteriology (Don et al. 2006).

\section{Preparation of Jatropha tanjorensis leaf extracts}

The extraction of $\mathrm{J}$. tanjorensis leaf was performed using absolute methanol, ethanol, hot and cold water as solvents. The extraction of the active ingredients of the plant leaf was performed using the method described by Harbone (1994) with slight modification. One hundred grams $(100 \mathrm{~g})$ of the pulverized leaves was Soxhlet extracted using $1000 \mathrm{~mL}$ of each solvent. The extracts were concentrated using a rotary evaporator (Resona, Germany) and were transferred into sterile bottles. The volatile oil obtained was purified by filtration using Whatman No.1 filter paper (Atata et al. 2003) and further sterilized by filtration through a millipore membrane filter of $0.45 \mu \mathrm{m}$ pore size (HAWP04700) (Merck, Darmstadt, Germany) (Sule and Agbabiaka 2008) and after that concentrated by evaporation using water bath at $42^{\circ} \mathrm{C}$. The sterile extracts were stored in sterile capped bottles and refrigerated at $4^{\circ} \mathrm{C}$ until use. The recovery rate of the extract was calculated using the formulae below;

\section{$\%$ Recovery of extract $=\mathrm{WA} / \mathrm{IW} \times 100$}

Where:

IW: Initial weight of extracts

WA: Weight of extracts recovered after extraction

\section{Microbial-free and sterility test of the extracts}

The extracts were tested for the presence or absence of turbidity using the Millipore filtration technique by introducing $2 \mathrm{~mL}$ of these extracts into $10 \mathrm{~mL}$ of sterile Mueller-Hinton broth and incubating at $37^{\circ} \mathrm{C}$ for $24 \mathrm{~h}$. The microbial-free extract was indicated by the absence of turbidity or clearness of the broth medium after the incubation (Sule and Agbabiaka 2008; Bodunrinde et al. 2020). Extracts were also tested for microbial growth and contaminants: extracts were re-dissolved in absolute ethanol. Then, the appropriate concentrations for bioassay analysis were made using sterile deionized distilled water and sterilized using a $0.45 \mu \mathrm{m}$ millipore membrane filter. Finally, one $\mathrm{mL}$ of each extract was inoculated onto nutrient agar to determine its sterility and incubated at $37^{\circ} \mathrm{C}$ for 24 hours. The absence of microbial growth in the extracts after incubation indicated that the extracts were sterile (Ashish et al. 2016).

\section{Phytochemical analysis of Jatropha tanjorensis crude extracts}

The phytochemical screening of the crude extracts was conducted using the slightly modified methods of Douye et al. (2013) and Paul et al. (2013).

\section{Bacterial inoculum standardization}

Bayode et al. (2021) modified the method to prepare the McFarland 0.5 turbidity standard to measure bacterial cell density. $0.5 \mathrm{~mL}$ of $1 \%(\mathrm{~W} / \mathrm{V})$ Barium chloride $\left(\mathrm{BaCl}_{2}\right)$ solution was added to $99.5 \mathrm{~mL}$ of $1 \%$ ( $\mathrm{vol} / \mathrm{vol}$ ) sulfuric acid $\left(\mathrm{H}_{2} \mathrm{SO}_{4}\right)$. McFarland standard tube was then sealed with Paraffin to prevent evaporation and stored in the dark at room temperature. The prepared McFarland standard's density accuracy was checked using a spectrophotometer 
with a $1 \mathrm{~cm}$ light path. The $0.5 \mathrm{McFarland}$ standard was vigorously agitated before use.

\section{Antibacterial assay of Jatropha tanjorensis crude extracts against bacteria isolated from wounds}

The antibacterial assay was determined by the agar diffusion method described by Morales-Cabrera et al. (2013). Bacterial isolates were cultivated in nutrient broth for 18 hours, and the bacterial suspension was prepared and compared to McFarland 0.5 turbidity standard. The bacterial suspension was inoculated onto solidified Mueller-Hinton agar surface by streaking with a sterile cotton-tipped swab to achieve a confluent growth. The inoculated plates were allowed to dry. Each extract $(2 \mathrm{~g})$ was dissolved in $10 \mathrm{~mL}$ dimethylsulfoxide (DMSO 30\%) to obtain a $200 \mathrm{mg} / \mathrm{mL}$ concentration of methanol, ethanol, and hot and cold water, respectively. Ciprofloxacin $(30 \mu \mathrm{g})$ was used as a positive control for all the bacterial isolates except Klebsiella pneumoniae. The positive control for $K$. pneumonia was chloramphenicol $(30 \mu \mathrm{g})$, while DMSO was the negative control. Next, $0.1 \mathrm{~mL}$ of each plant extract was dropped onto a $10 \mathrm{~mm}$ filter paper disc and allowed to dry in the incubator at $45{ }^{\circ} \mathrm{C}$ for 15 minutes. The paper discs were then impregnated on the surface of the inoculated nutrients agar plate using a dispenser and incubated for $24 \mathrm{hrs}, 48 \mathrm{hrs}$, and $72 \mathrm{hrs}$ at $37^{\circ} \mathrm{C}$. The zones of inhibition were measured with a vernier caliper (Mitutoyo 530-119) (Cranbury, New Jersey, United States) at every $24 \mathrm{hrs}$ interval. The analytical grade solvents used for the extraction were used as control experiments (CLSI 2014).

\section{Determination of Minimum Inhibitory Concentration (MIC) of Jatropha tanjorensis crude extracts against bacteria isolated from wounds}

The initial concentration of leaf extracts $(200 \mathrm{mg} / \mathrm{mL})$ was diluted using double-fold serial dilution by transferring $5 \mathrm{~mL}$ of the sterile leaf extract (stock solution) into $5 \mathrm{~mL}$ of sterile nutrient broth to obtain $100 \mathrm{mg} / \mathrm{mL}$ concentration. That was repeated to obtain subsequent dilutions such as 50 $\mathrm{mg} / \mathrm{mL}, 25 \mathrm{mg} / \mathrm{mL}, 12.5 \mathrm{mg} / \mathrm{mL}, 6.25 \mathrm{mg} / \mathrm{mL}$, and 3.125 $\mathrm{mg} / \mathrm{mL}$ (Ibekwe et al. 2001). Each concentration was inoculated with $0.1 \mathrm{~mL}$ of the standardized bacterial cell suspension and incubated at $37^{\circ} \mathrm{C}$ for 24 hours. The turbidity or cloudiness of the broth indicated the growth of the bacterial inoculum in the broth medium. The lowest concentration of extract that inhibited bacterial isolates' growth was considered the Minimum Inhibitory Concentration (MIC).

\section{Determination of Jatropha tanjorensis crude extracts bioactive compounds}

The crude extracts of $J$. tanjorensis were analyzed to determine the chemical constituents using a Varian 4000 GC-MS system equipped with an HP-5MS capillary column $(30 \mathrm{~m} \times 0.25 \mathrm{~mm} \times 0.25 \mu \mathrm{m})$ with a running time of 40 minutes. In addition, an Agilent column, HP-5MS capillary column $(30 \mathrm{~m} \times 0.25 \mathrm{~mm} \times 0.25 \mu \mathrm{m})$ was also utilized (Colombini et al. 2010). The particular compounds present in the extract of the leaves were identified by relating their mass spectral fragmentation patterns of the respective peaks in the chromatogram with those stored in the National Institute of Standards and Technology Mass Spectral database (NIST 1998) library.

\section{Statistical analysis}

Data were analyzed using SPSS 16.0 for the windows evaluation version by using analysis of variance (ANOVA) and Duncan's Multiple Range Test to estimate means. $\mathrm{P}<0.05$ was considered significantly different.

\section{RESULTS AND DISCUSSION}

\section{Percentage of bacterial isolate on wound samples}

Coagulase-positive $S$. aureus was present in the highest percentage in all wound samples compared to other isolates. For example, in the crushed leg injury, S. aureus was present at $100 \%$, while S. pyogenes and Proteus mirabilis were found to have the least at $1(3 \%)$ and 2 (4\%), correspondingly as shown in Table 1 .

\section{The percentage recovery of Jatropha tanjorensis crude extracts}

Methanol extract of $J$. tanjorensis had the highest percentage recovery $(82 \%)$, while cold water extract had the lowest percentage (53\%), as shown in Table 2.

\section{Qualitative and quantitative phytochemical compounds of Jatropha tanjorensis leaf extracts}

Bioactive compounds of $J$. tanjorensis leaf extracts in different solvents varied, as presented in Table 3. The results of the quantitative analysis of phytochemical compounds are presented in Table 4. It showed that saponin content in all solvents was high compared to other compounds, and the highest was in the methanol extract $(57.72 \mathrm{mg} / \mathrm{g})$. Flavonoid content had the lowest percentage of all extracts, and the highest flavonoid content was obtained in the methanol extract $(2 \mathrm{mg} / \mathrm{g})$. There were significant differences between the mean values of quantitative phytochemical analysis of extracts at $\mathrm{p}<0.05$.

\section{Gas Chromatography-Mass Spectrophotometer (GCMS) profile of Jatropha tanjorensis crude extracts}

The GCMS analysis of J. tanjorensis showed that all extracts contained Octadecanoic acid, n-Hexadecanoic acid, and phytol. The methyl ester was obtained in the methanol and ethanol extracts. Eicosane was extracted from methanol extract only, while ethanol extract revealed the presence of 6-Octadecanoic acid. In addition, oleic acid was extracted from hot water and cold water. Hot water also revealed the presence of vitamin $E$, while hydroquinone was only obtained in the cold-water extract of $J$. tanjorensis, as shown in Table 5. 


\section{Antibacterial activities of Jatropha tanjorensis leaf crude extract}

The antibacterial activities of $J$. tanjorensis leaf extract against Gram-positive and Gram-negative bacteria are shown in Tables $6 \mathrm{a}$ and $6 \mathrm{~b}$, respectively. The methanol, ethanol, hot water, and cold-water extracts of J. tanjorensis leaf inhibited the growth of $S$. aureus coagulase positive from all the wound samples at $200 \mathrm{mg} / \mathrm{mL}$ concentration. Staphylococcus aureus coagulase positive had the highest Zone of Inhibition (ZOI) of $33 \mathrm{~mm}$, while the lowest ZOI $(5 \mathrm{~mm})$ was obtained from cold water extract. Methanol extract had the highest ZOI ranging from $16 \mathrm{~mm}$ to $17 \mathrm{~mm}$ against $E$. coli, while the lowest inhibitory zone against $E$. coli was obtained from cold water extract (3-4 mm).

Minimum Inhibitory Concentration ( $\mathrm{mg} / \mathrm{mL})$ of methanol leaf extracts of Jatropha tanjorensis against bacterial isolates from wound samples

The MIC of the methanol extracts of J. tanjorensis on the isolated bacteria is shown in Table 7, ranging from 12.5 to $100 \mathrm{mg} / \mathrm{mL}$.

Table 2. Percentage of leaf extracts of Jatropha tanjorensis

\begin{tabular}{lccc}
\hline Extracts & $\begin{array}{c}\text { Weight of } \\
\text { leaves } \\
(\mathbf{O W})(\mathbf{g})\end{array}$ & $\begin{array}{c}\text { Weight of } \\
\text { extract } \\
(\mathbf{E W})(\mathbf{g})\end{array}$ & $\begin{array}{c}\text { Percentage of } \\
\text { extract } \\
(\boldsymbol{\%})\end{array}$ \\
\hline Methanol & 100 & 82.01 & 82 \\
Ethanol & 100 & 79.50 & 79.50 \\
Hot water & 100 & 56.36 & 56.4 \\
Cold water & 100 & 53.04 & 53 \\
\hline
\end{tabular}

Table 1. Percentage occurrence of bacteria from wound samples

\begin{tabular}{|c|c|c|c|c|c|c|c|c|}
\hline $\begin{array}{l}\text { Wound } \\
\text { types }\end{array}$ & $\begin{array}{l}\text { S. aureus } \\
\text { Coag +ve }\end{array}$ & $\begin{array}{c}\text { S. aureus } \\
\text { Coag -ve }\end{array}$ & E. coli & P. aeruginosa & K. pneumoniae & P. mirabilis & S. pyogenes & SWG \\
\hline \multicolumn{9}{|c|}{ Number/ $(\%)$} \\
\hline$\overline{\mathrm{DMFU}}$ & $14(57)$ & $4(21)$ & $2(4)$ & $0(0)$ & $1(3)$ & $1(3)$ & $0(0)$ & $3(12)$ \\
\hline BW & $11(56)$ & $3(12)$ & $2(4)$ & $2(4)$ & $2(4)$ & $2(4)$ & $2(4)$ & $3(12)$ \\
\hline PSD & $9(18)$ & $0(0)$ & $0(0)$ & $0(0)$ & $0(0)$ & $0(0)$ & $0(0)$ & $41(82)$ \\
\hline AW & $31(76)$ & $6(12)$ & $2(4)$ & $2(4)$ & $2(4)$ & $0(0)$ & $0(0)$ & $0(0)$ \\
\hline CLU & $13(64)$ & $5(15)$ & $3(9)$ & $2(6)$ & $2(6)$ & $0(0)$ & $0(0)$ & $0(0)$ \\
\hline CALU & $11(60)$ & $3(9)$ & $6(12)$ & $3(6)$ & $3(6)$ & $0(0)$ & $2(8)$ & $0(0)$ \\
\hline SCLU & $12(58)$ & $0(0)$ & $6(12)$ & $6(12)$ & $0(0)$ & $6(12)$ & $0(0)$ & $0(0)$ \\
\hline $\mathrm{CHI}$ & $10(50)$ & $0(0)$ & $5(25)$ & $5(25)$ & $0(0)$ & $0(0)$ & $0(0)$ & $0(0)$ \\
\hline CLI & $10(100)$ & $0(0)$ & $0(0)$ & $0(0)$ & $0(0)$ & $0(0)$ & $0(0)$ & $0(0)$ \\
\hline
\end{tabular}

Note: DMFU: Diabetes Mellitus foot ulcer wounds; BW: Burn wounds; PSD: Post skin graft wound dehiscence; AW: Avulsion wounds; CLU: Chronic leg ulcer wounds; CALU: Chronic arterial leg ulcer wounds; SCLU: Sickle cell leg ulcer wounds; CHI: Crushed hand injury; CLI: Crushed leg injury; SWG: Samples with no growth; N: Number of bacterial isolates; \%: percentage occurrence. $1^{\text {st. }}$ number indicated the number of occurrences. $2^{\text {nd. }}$ number indicated the percentage of occurrence
Table 3. The qualitative phytochemical compound of Jatropha tanjorensis leaf extracts

\begin{tabular}{lcccc}
\hline Phytochemicals & Methanol & Ethanol & $\begin{array}{c}\text { Hot } \\
\text { water }\end{array}$ & $\begin{array}{c}\text { Cold } \\
\text { water }\end{array}$ \\
\hline Saponin & + & + & + & + \\
Tannin & + & + & + & + \\
Phlobatannin & - & - & - & - \\
Flavonoid & + & + & + & + \\
Steroid & + & + & - & - \\
Terpenoid & + & + & + & + \\
Alkaloid & - & - & - & - \\
Anthraquinone & - & - & - & - \\
Cardiac glycosides & $\mathrm{NA}$ & $\mathrm{NA}$ & $\mathrm{NA}$ & $\mathrm{NA}$ \\
Legal test & + & + & + & + \\
Keller kiliani test & + & + & + & + \\
Salkwoski test & + & + & + & + \\
Lieberman test & + & + & - & - \\
\hline
\end{tabular}

Note: + : positive; - : negative NA: Not applicable

Table 4. The quantitative phytochemical compound of Jatropha tanjorensis leaf extracts

\begin{tabular}{|c|c|c|c|c|}
\hline $\begin{array}{c}\text { Phyto- } \\
\text { chemical } \\
(\mathrm{mg} / \mathrm{g})\end{array}$ & Methanol & Ethanol & Hot water & Coldwater \\
\hline Tannin & $8.33 \pm 0.01^{\mathrm{d}}$ & $5.32 \pm 0.03^{c}$ & $4.01 \pm 0.01^{b}$ & $2.55 \pm 0.01^{\mathrm{a}}$ \\
\hline Saponin & $57.72 \pm 0.39^{d}$ & $43.91 \pm 0.39^{c}$ & $11.36 \pm 0.39^{b}$ & $3.55 \pm 0.39^{\mathrm{a}}$ \\
\hline Flavonoid & $2.14 \pm 0.01^{\mathrm{d}}$ & $1.56 \pm 0.01^{\mathrm{c}}$ & $1.44 \pm 0.01^{\mathrm{b}}$ & $0.96 \pm 0.01^{\mathrm{a}}$ \\
\hline Terpenoid & $24.74 \pm 0.07^{\mathrm{d}}$ & $19.14 \pm 0.06^{c}$ & $15.70 \pm 0.06^{b}$ & $12.25 \pm 0.06^{\mathrm{a}}$ \\
\hline Glycosides & $23.44 \pm 0.06^{\mathrm{d}}$ & $18.38 \pm 0.07^{\mathrm{c}}$ & $15.45 \pm 0.07^{b}$ & $12.43 \pm 0.07^{\mathrm{a}}$ \\
\hline Steroid & $6.71 \pm 0.03^{\mathrm{c}}$ & $5.15 \pm 0.01^{b}$ & $0.00 \pm 0.00^{\mathrm{a}}$ & $0.00 \pm 0.00^{\mathrm{a}}$ \\
\hline
\end{tabular}


Table 5. Identified chemical compounds in Jatropha tanjorensis extracts by GC-MS analysis

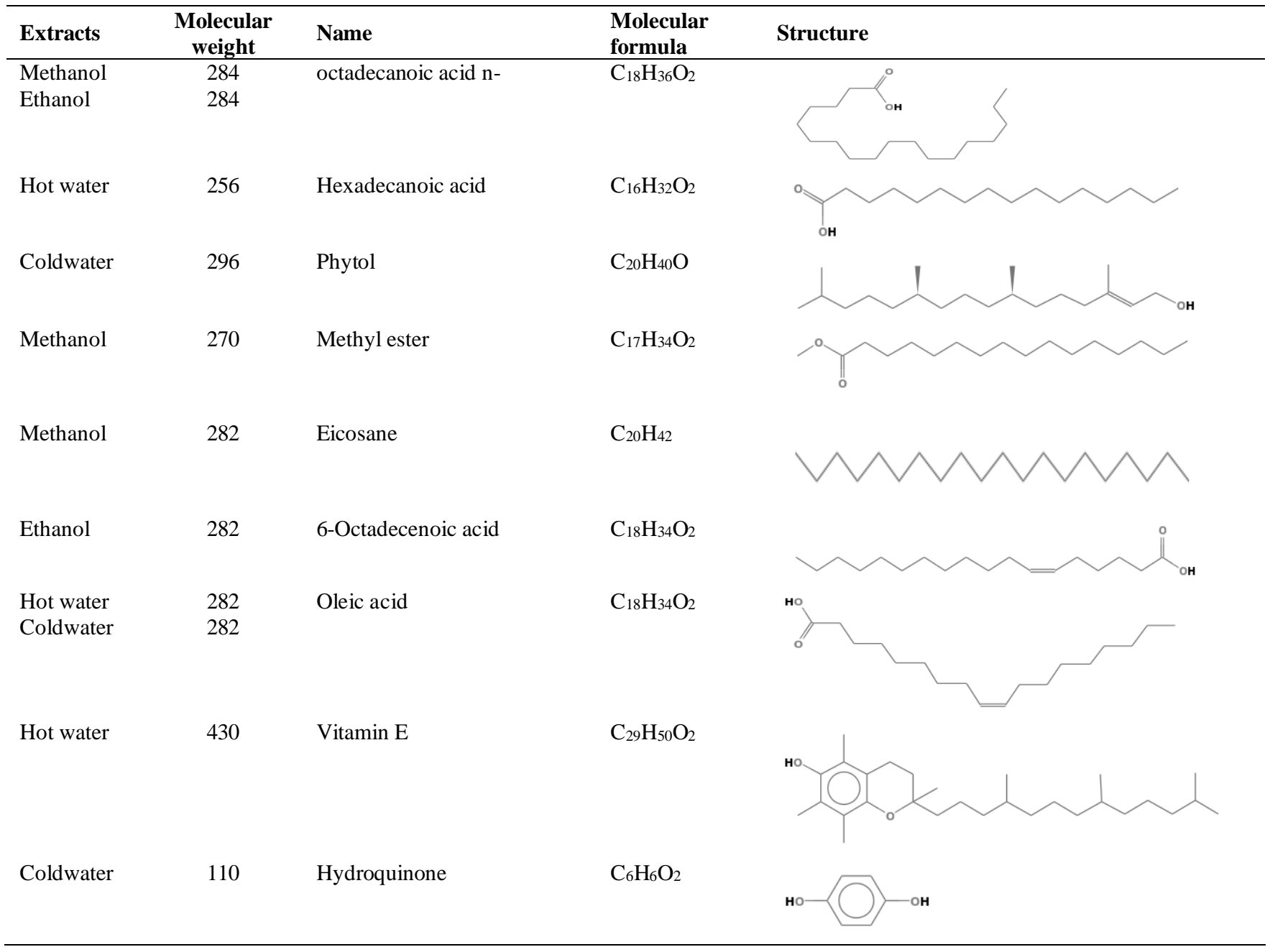

Table 6A. Diameter of inhibitory zone of Jatropha tanjorensis leaf extracts against Gram-positive bacteria isolated from wound samples

\begin{tabular}{|c|c|c|c|c|c|c|c|}
\hline Isolates & $\begin{array}{c}\text { Wound } \\
\text { types }\end{array}$ & Ethanol & Methanol & Hot water & Coldwater & $\begin{array}{l}\text { Positive } \\
\text { control }\end{array}$ & $\begin{array}{l}\text { Negative } \\
\text { control }\end{array}$ \\
\hline Streptococcus & DMFU & $22.67 \pm 0.57^{\mathrm{a}}$ & $33.00 \pm 0.00^{\mathrm{a}}$ & $12.67 \pm 0.57^{\mathrm{a}}$ & $7.67 \pm 0.57^{\mathrm{a}}$ & $31.67 \pm 0.57^{\mathrm{a}}$ & $00.00 \pm 0.00^{\mathrm{a}}$ \\
\hline pyogenes & BW & $22.33 \pm 0.57^{\mathrm{ab}}$ & $32.33 \pm 0.57^{\mathrm{b}}$ & $12.00 \pm 0.00^{\mathrm{b}}$ & $7.33 \pm 0.57^{\mathrm{ab}}$ & $30.33 \pm 0.28^{b}$ & $00.00 \pm 0.00^{\mathrm{a}}$ \\
\hline Coagulase positive & DMFU & $23.67 \pm 0.57^{\mathrm{ab}}$ & $32.33 \pm 0.57^{\mathrm{a}}$ & $14.33 \pm 0.57^{\mathrm{ab}}$ & $6.00 \pm 0.00^{\mathrm{a}}$ & $31.50 \pm 0.87^{\mathrm{a}}$ & $00.00 \pm 0.00^{\mathrm{a}}$ \\
\hline \multirow{8}{*}{ Staphylococcus aureus } & BW & $24.00 \pm 0.00^{\mathrm{ac}}$ & $27.67 \pm 0.57^{\mathrm{ab}}$ & $14.67 \pm 0.57^{\mathrm{a}}$ & $6.33 \pm 0.57^{\mathrm{ab}}$ & $26.50 \pm 1.00^{\mathrm{c}}$ & $00.00 \pm 0.00^{\mathrm{a}}$ \\
\hline & PSD & $24.67 \pm 0.57^{\mathrm{b}}$ & $33.00 \pm 0.00^{\mathrm{ac}}$ & $13.33 \pm 0.57^{b c}$ & $6.67 \pm 0.57^{b}$ & $32.67 \pm 0.29^{b}$ & $00.00 \pm 0.00^{\mathrm{a}}$ \\
\hline & AW & $23.00 \pm 0.00^{\mathrm{c}}$ & $26.67 \pm 0.57^{\mathrm{bc}}$ & $12.67 \pm 0.57^{\mathrm{ab}}$ & $5.67 \pm 0.57^{\mathrm{a}}$ & $25.17 \pm 0.76^{\mathrm{ab}}$ & $00.00 \pm 0.00^{\mathrm{a}}$ \\
\hline & CLU & $23.67 \pm 0.57^{\mathrm{a}}$ & $33.00 \pm 0.00^{\mathrm{ab}}$ & $14.67 \pm 0.57^{\mathrm{c}}$ & $5.00 \pm 0.00^{\mathrm{ab}}$ & $32.67 \pm 0.58^{\mathrm{ac}}$ & $00.00 \pm 0.00^{\mathrm{a}}$ \\
\hline & CALU & $22.33 \pm 0.57^{\mathrm{ab}}$ & $27.33 \pm 0.57^{\mathrm{a}}$ & $14.33 \pm 0.57^{\mathrm{b}}$ & $6.33 \pm 0.57^{\mathrm{ac}}$ & $25.17 \pm 0.76^{\mathrm{bc}}$ & $00.00 \pm 0.00^{\mathrm{a}}$ \\
\hline & SCLU & $24.00 \pm 0.00^{\mathrm{ab}}$ & $26.67 \pm 0.57^{\mathrm{ab}}$ & $13.67 \pm 0.57^{\mathrm{a}}$ & $6.33 \pm 0.57^{c}$ & $24.50 \pm 0.50^{\mathrm{a}}$ & $00.00 \pm 0.00^{\mathrm{a}}$ \\
\hline & $\mathrm{CHI}$ & $23.00 \pm 0.00^{\mathrm{a}}$ & $32.33 \pm 0.57^{\mathrm{a}}$ & $14.67 \pm 0.57^{\mathrm{ab}}$ & $5.33 \pm 0.57^{\mathrm{ab}}$ & $31.33 \pm 1.15^{\mathrm{ab}}$ & $00.00 \pm 0.00^{\mathrm{a}}$ \\
\hline & CLI & $24.33 \pm 0.57^{\mathrm{bc}}$ & $30.00 \pm 0.00^{\mathrm{ab}}$ & $13.33 \pm 0.57^{\mathrm{a}}$ & $6.33 \pm 0.57^{\mathrm{a}}$ & $28.17 \pm 0.29^{\mathrm{a}}$ & $00.00 \pm 0.00^{\mathrm{a}}$ \\
\hline Coagulase negative & DMFU & $22.33 \pm 0.57^{\mathrm{a}}$ & $28.33 \pm 0.57^{b}$ & $12.33 \pm 0.57^{\mathrm{a}}$ & $7.67 \pm 0.57^{\mathrm{a}}$ & $26.50 \pm 0.87^{b c}$ & $00.00 \pm 0.00^{\mathrm{a}}$ \\
\hline \multirow[t]{4}{*}{ Staphylococcus aureus } & BW & $24.00 \pm 0.00^{\mathrm{ab}}$ & $30.00 \pm 0.00^{\mathrm{a}}$ & $12.00 \pm 0.00^{\mathrm{ab}}$ & $7.33 \pm 0.57^{\mathrm{ab}}$ & $28.83 \pm 0.29^{\mathrm{ab}}$ & $00.00 \pm 0.00^{\mathrm{a}}$ \\
\hline & PSD & $22.33 \pm 0.57^{\mathrm{a}}$ & $28.33 \pm 0.57^{\mathrm{bc}}$ & $14.00 \pm 0.00^{\mathrm{a}}$ & $6.67 \pm 0.57^{\mathrm{a}}$ & $26.67 \pm 0.29^{\mathrm{a}}$ & $00.00 \pm 0.00^{\mathrm{a}}$ \\
\hline & AW & $22.33 \pm 0.57^{\mathrm{bc}}$ & $30.00 \pm 0.00^{\mathrm{a}}$ & $12.67 \pm 0.57^{\mathrm{ab}}$ & $7.00 \pm 0.00^{c}$ & $26.67 \pm 0.29^{\mathrm{ab}}$ & $00.00 \pm 0.00^{\mathrm{a}}$ \\
\hline & CLU & $23.67 \pm 0.57^{\mathrm{a}}$ & $28.66 \pm 0.57^{\mathrm{ab}}$ & $12.67 \pm 0.57^{\mathrm{ac}}$ & $7.67 \pm 0.57^{b c}$ & $24.83 \pm 0.29^{\mathrm{a}}$ & $00.00 \pm 0.00^{\mathrm{a}}$ \\
\hline
\end{tabular}

Note: DMFU: Diabetes Mellitus foot ulcer wounds; BW: Burn wounds; PSD: Post skin graft wound dehiscence; AW: Avulsion wounds; CLU: Chronic leg ulcer wounds; CALU: Chronic arterial leg ulcer wounds; SCLU: Sickle cell leg ulcer wounds; CHI: Crushed hand injury; CLI: Crushed leg injury; Positive control: Ciprofloxacin $(30 \mu \mathrm{g})$, Negative control: Dimethyl sulfoxide (DMSO). The result represents the mean of triplicate values expressed in mean \pm standard error with a significant difference at $p<0.05$. 
Table 6B. Diameter of inhibitory zone of Jatropha tanjorensis leaf extracts against Gram-negative bacteria isolated wound samples

\begin{tabular}{|c|c|c|c|c|c|c|c|}
\hline Isolates & $\begin{array}{c}\text { Wound } \\
\text { types }\end{array}$ & Methanol & Ethanol & Hot water & Coldwater & $\begin{array}{l}\text { Positive } \\
\text { control }\end{array}$ & $\begin{array}{l}\text { Negative } \\
\text { control }\end{array}$ \\
\hline \multirow[t]{7}{*}{ Escherichia coli } & DMFU & $16.67 \pm 0.17^{\mathrm{a}}$ & $9.67 \pm 0.33^{\mathrm{ab}}$ & $6.33 \pm 0.33^{\mathrm{a}}$ & $3.83 \pm 0.17^{\mathrm{ab}}$ & $17.83 \pm 0.17^{\mathrm{a}}$ & $00.00 \pm 0.00^{\mathrm{a}}$ \\
\hline & BW & $16.33 \pm 0.33^{\mathrm{ab}}$ & $12.00 \pm 0.17^{\mathrm{b}}$ & $6.50 \pm 0.29^{\mathrm{bc}}$ & $3.83 \pm 0.17^{\mathrm{bc}}$ & $17.50 \pm 0.33^{\mathrm{ab}}$ & $00.00 \pm 0.00^{\mathrm{a}}$ \\
\hline & PSD & $17.00 \pm 0.00^{\mathrm{a}}$ & $10.33 \pm 0.17^{\mathrm{ab}}$ & $5.67 \pm 0.33^{\mathrm{a}}$ & $3.67 \pm 0.17^{\mathrm{b}}$ & $17.83 \pm 0.17^{\mathrm{b}}$ & $00.00 \pm 0.00^{\mathrm{a}}$ \\
\hline & AW & $16.83 \pm 0.33^{\mathrm{ab}}$ & $11.83 \pm 0.17^{\mathrm{a}}$ & $5.33 \pm 0.33^{b}$ & $3.83 \pm 0.17^{\mathrm{a}}$ & $17.33 \pm 0.33^{\mathrm{ab}}$ & $00.00 \pm 0.00^{\mathrm{a}}$ \\
\hline & CLU & $17.00 \pm 0.00^{\mathrm{b}}$ & $10.33 \pm 0.00^{\mathrm{bc}}$ & $6.33 \pm 0.17^{\mathrm{ac}}$ & $3.00 \pm 0.00^{\mathrm{ac}}$ & $17.67 \pm 0.17^{\mathrm{ac}}$ & $00.00 \pm 0.00^{\mathrm{a}}$ \\
\hline & CALU & $17.00 \pm 0.29^{\mathrm{ab}}$ & $12.00 \pm 0.17^{\mathrm{ab}}$ & $6.67 \pm 0.33^{\mathrm{ab}}$ & $4.00 \pm 0.00^{\mathrm{a}}$ & $17.67 \pm 0.29^{b}$ & $00.00 \pm 0.00^{\mathrm{a}}$ \\
\hline & SCLU & $16.67 \pm 0.17^{\mathrm{a}}$ & $10.33 \pm 0.17^{\mathrm{ab}}$ & $6.00 \pm 0.00^{\mathrm{a}}$ & $3.83 \pm 0.17^{\mathrm{ab}}$ & $17.67 \pm 0.17^{\mathrm{a}}$ & $00.00 \pm 0.00^{\mathrm{a}}$ \\
\hline \multirow{6}{*}{$\begin{array}{l}\text { Pseudomonas } \\
\text { aeruginosa }\end{array}$} & DMFU & $20.33 \pm 0.33^{\mathrm{ab}}$ & $15.83 \pm 0.17^{\mathrm{ac}}$ & $9.67 \pm 0.33^{\mathrm{bc}}$ & $4.00 \pm 0.00^{\mathrm{bc}}$ & $30.33 \pm 0.33^{\mathrm{ab}}$ & $00.00 \pm 0.00^{\mathrm{a}}$ \\
\hline & $\mathrm{BW}$ & $22.00 \pm 0.00^{\mathrm{bc}}$ & $15.67 \pm 0.17^{\mathrm{ab}}$ & $8.33 \pm 0.33^{\mathrm{a}}$ & $4.83 \pm 0.17^{\mathrm{a}}$ & $31.33 \pm 0.33^{\mathrm{bc}}$ & $00.00 \pm 0.00^{\mathrm{a}}$ \\
\hline & PSD & $20.67 \pm 0.33^{b}$ & $15.00 \pm 0.00^{\mathrm{b}}$ & $8.00 \pm 0.00^{\mathrm{ab}}$ & $4.17 \pm 0.17^{\mathrm{ab}}$ & $30.00 \pm 0.00^{\mathrm{ac}}$ & $00.00 \pm 0.00^{\mathrm{a}}$ \\
\hline & AW & $21.67 \pm 0.33^{\mathrm{ac}}$ & $14.67 \pm 0.33^{\mathrm{a}}$ & $9.00 \pm 0.00^{\mathrm{a}}$ & $5.00 \pm 0.17^{\mathrm{a}}$ & $31.33 \pm 0.33^{\mathrm{a}}$ & $00.00 \pm 0.00^{\mathrm{a}}$ \\
\hline & CLU & $20.00 \pm 0.00^{\mathrm{a}}$ & $16.00 \pm 0.00^{\mathrm{ab}}$ & $9.67 \pm 0.33^{b}$ & $4.33 \pm 0.17^{b}$ & $30.33 \pm 0.33^{\mathrm{a}}$ & $00.00 \pm 0.00^{\mathrm{a}}$ \\
\hline & CALU & $20.67 \pm 0.33^{\mathrm{ab}}$ & $15.33 \pm 0.33^{\mathrm{b}}$ & $8.00 \pm 0.00^{\mathrm{ab}}$ & $5.00 \pm 0.00^{\mathrm{ab}}$ & $30.67 \pm 0.33^{b}$ & $00.00 \pm 0.00^{\mathrm{a}}$ \\
\hline \multirow[t]{5}{*}{ Proteus mirabilis } & DMFU & $24.00 \pm 0.00^{\mathrm{b}}$ & $15.83 \pm 0.17^{\mathrm{a}}$ & $12.17 \pm 0.17^{\mathrm{a}}$ & $6.33 \pm 0.33^{\mathrm{a}}$ & $27.00 \pm 0.00^{\mathrm{bc}}$ & $00.00 \pm 0.00^{\mathrm{a}}$ \\
\hline & $\mathrm{BW}$ & $28.17 \pm 0.17^{\mathrm{ab}}$ & $15.67 \pm 0.17^{\mathrm{ab}}$ & $12.00 \pm 0.00^{\mathrm{ab}}$ & $6.50 \pm 0.29^{\mathrm{bc}}$ & $30.83 \pm 0.17^{\mathrm{ab}}$ & $00.00 \pm 0.00^{\mathrm{a}}$ \\
\hline & PSD & $28.17 \pm 0.17^{\mathrm{a}}$ & $15.00 \pm 0.00^{\mathrm{ac}}$ & $12.67 \pm 0.33^{\mathrm{ac}}$ & $5.67 \pm 0.33^{\mathrm{ab}}$ & $31.33 \pm 0.17^{\mathrm{a}}$ & $00.00 \pm 0.00^{\mathrm{a}}$ \\
\hline & AW & $28.33 \pm 0.33^{\mathrm{b}}$ & $14.67 \pm 0.33^{\mathrm{a}}$ & $12.00 \pm 0.00^{\mathrm{bc}}$ & $5.33 \pm 0.17^{\mathrm{a}}$ & $30.33 \pm 0.17^{\mathrm{c}}$ & $00.00 \pm 0.00^{\mathrm{a}}$ \\
\hline & CLU & $24.00 \pm 0.00^{c}$ & $16.00 \pm 0.00^{\mathrm{bc}}$ & $10.00 \pm 0.00^{\mathrm{a}}$ & $6.33 \pm 0.00^{\mathrm{ab}}$ & $27.33 \pm 0.33^{\mathrm{b}}$ & $00.00 \pm 0.00^{\mathrm{a}}$ \\
\hline \multirow[t]{3}{*}{ Klebsiella pneumoniae } & DMFU & $22.00 \pm 0.00^{\mathrm{a}}$ & $14.00 \pm 0.00^{\mathrm{ab}}$ & $8.00 \pm 0.00^{\mathrm{a}}$ & $4.00 \pm 0.00^{\mathrm{ab}}$ & $24.00 \pm 0.00^{\mathrm{a}}$ & $00.00 \pm 0.00^{\mathrm{a}}$ \\
\hline & $\mathrm{BW}$ & $24.83 \pm 0.17^{\mathrm{ab}}$ & $12.67 \pm 0.33^{b c}$ & $7.33 \pm 0.33^{\mathrm{ab}}$ & $3.67 \pm 0.17^{\mathrm{a}}$ & $27.33 \pm 0.17^{\mathrm{ab}}$ & $00.00 \pm 0.00^{\mathrm{a}}$ \\
\hline & PSD & $22.00 \pm 0.00^{\mathrm{bc}}$ & $12.67 \pm 0.33^{\mathrm{a}}$ & $7.00 \pm 0.00^{\mathrm{a}}$ & $3.67 \pm 0.17^{\mathrm{c}}$ & $24.33 \pm 0.33^{\mathrm{bc}}$ & $00.00 \pm 0.00^{\mathrm{a}}$ \\
\hline
\end{tabular}

Note: DMFU: Diabetes Mellitus foot ulcer wounds); BW: Burn wounds; PSD: Post skin graft wound dehiscence; AW: Avulsion wounds; CLU: Chronic leg ulcer wounds; CALU: Chronic arterial leg ulcer wounds; SCLU: Sickle cell leg ulcer wounds; CHI: Crushed hand injury; CLI: Crushed leg injury; Positive control: Ciprofloxacin $(30 \mu \mathrm{g})$, Negative control: Dimethyl sulfoxide (DMSO). The result represents the mean of triplicate values expressed in mean \pm standard error with a significant difference at $\mathrm{p}<0.05$.

Table 7. Minimum Inhibitory Concentration $(\mathrm{mg} / \mathrm{mL})$ of methanol leaf extracts of Jatropha tanjorensis against bacteria isolated from wounds

\begin{tabular}{lccccccc}
\hline Wounds & $\begin{array}{c}\text { S. aureus Coag. } \\
+\mathbf{v e} \\
(\mathbf{m g} / \mathbf{m L})\end{array}$ & $\begin{array}{c}\text { S. aureus } \\
\text { Coag. }-\mathbf{v e} \\
(\mathbf{m g} / \mathbf{m L})\end{array}$ & $\begin{array}{c}\text { E. coli } \\
(\mathbf{m g} / \mathbf{m L})\end{array}$ & $\begin{array}{c}\boldsymbol{P .} \text { aeruginosa } \\
(\mathbf{m g} / \mathbf{m L})\end{array}$ & $\begin{array}{c}\boldsymbol{P} \text {. mirabilis } \\
(\mathbf{m g} / \mathbf{m L})\end{array}$ & $\begin{array}{c}\text { K. pneumoniae } \\
(\mathbf{m g} / \mathbf{m L})\end{array}$ & $\begin{array}{c}\text { S. pyogenes } \\
(\mathbf{m g} / \mathbf{m L})\end{array}$ \\
\hline DMFU & 12.5 & 25 & 50 & NA & 100 & 50 & NA \\
BW & 25 & 12.5 & 100 & 100 & 50 & 50 & 12.5 \\
PSD & 12.5 & NA & NA & NA & NA & NA & NA \\
AW & 25 & 25 & 100 & 100 & 50 & NA & NA \\
CLU & 12.5 & 12.5 & 50 & 100 & 50 & NA & NA \\
CALU & 25 & 25 & 100 & 100 & 100 & NA & NA \\
SCLU & 25 & NA & 100 & 100 & NA & 50 & 12.5 \\
CHI & 12.5 & NA & 50 & 100 & NA & NA & NA \\
CLI & 12.5 & NA & NA & NA & NA & NA & NA \\
\hline
\end{tabular}

Note: DMFU: Diabetes Mellitus foot ulcer wounds; BW: Burn wounds; PSD: Post skin graft wound dehiscence; AW: Avulsion wounds; CLU: Chronic leg ulcer wounds; CALU: Chronic arterial leg ulcer wounds; SCLU: Sickle cell leg ulcer wounds; CHI: Crushed hand injury; CLI: Crushed leg injury; Positive control: Ciprofloxacin $(30 \mu \mathrm{g})$, Negative control: Dimethyl sulfoxide (DMSO). NA: Not available, Coag. +ve: Coagulase positive, Coag. -ve: Coagulase-negative, Values presented in the table were the average of triplicate experiments

\section{Discussions}

This study showed that bacteria isolates were present in all wound samples. A previous study by Gautam et al. (2013) reported that $60 \%$ of pus culture was positive for bacterial growth, including; S. aureus, $P$. aeruginosa, $S$. pyogenes, K. pneumoniae, and Proteus species. A study by Meenakshi et al. (2015) showed the presence of several bacteria, i.e., S. aureus 38 (29.6\%), S. pyogenes 8 (6.2\%), P. aeruginosa $28(21.8 \%)$, E. coli $26(20.3 \%)$, and $K$. pneumoniae $2(1.5 \%)$. Thomsen et al. (2010) observed the presence of a high percentage of coagulase-positive $S$. aureus from chronic venous leg ulcers, similar to the results of this study. Ogba et al. (2014) reported that burn wound samples collected from public hospitals in Calabar, Nigeria, infected by $S$. aureus as the most prevalent bacteria (30.8\%), followed by P. aeruginosa $(17.3 \%)$ and Streptococcus spp. were being the lowest (1.3\%), which was in agreement with the findings of this study.

The high yield percentage of $J$. tanjorensis methanol extract is similar to the findings of Beni et al. (2014) on 
methanol leaf extracts of $J$. curcas. The high yield of $J$. tanjorensis methanol extract could be due to its polarity as an extraction solvent. Methanol is a universal solvent that dissolves all compounds, i.e., polar, semi-polar, or nonpolar. In the extraction process, the composition, color, aroma, and extract yield are influenced by the raw material's type, size, maturity level, type of solvent, temperature, extraction time, and extraction method (Farrel 1990). In addition, the percentage of secondary metabolites in the methanol extract might be strongly influenced by the plant leaves used for extraction, which is generally less than 10\% (Van Beek 1999).

A study by Omoregie and Osagie (2007) on the phytochemical analysis of $J$. tanjorensis revealed that it contains biochemical compounds such as alkaloids, flavonoids, tannins, cardiac glycosides, anthraquinones, and saponins which are similar to the findings in this study. The results of GC-MS analysis of J. tanjorensis crude extracts in this study were in line with the results of Ebana et al. (2019), which identified the presence of a total of 13 compounds, specifically octadecanoic acid, 2-hydroxy-1(hydroxymethyl)-ethyl ester, n-hexadecanoic acid, octadecanoic acid, and propanoic acid. Ebana et al. (2019) also reported the presence of three flavonoids, three amines, two associated hydrocarbons, two alkanes, and two alkaloids. Flavonoids and alkaloids were also detected in the results of GC-MS analysis.

The high inhibitory effect of $J$. tanjorensis leaf extracts on bacterial isolates could be due to the chemical compounds with wound-healing potency in the plant, as Hartwell reported earlier (1969). Furthermore, Viswanathan et al. (2012) reported that $J$. tanjorensis extracts exhibit potent anti-inflammatory and antioxidant activities contributing to its wound healing properties, which could also be a reason for the high activity index of $J$. tanjorensis crude extract observed in this study. So, antioxidant properties in J. tanjorensis crude extracts might play a role in reducing complicated/adverse effects. Therefore, antioxidant properties seem crucial in successfully treating wounds (Houghton et al. 2005).

This study revealed that the methanol extract of $J$. tanjorensis had the highest inhibitory activity against bacteria-infected wounds compared to other crude extracts and commercial antibiotics. It might be due to several bioactive chemical compounds that are synergistically active and increase the antibacterial activity of $J$. tanjorensis crude extract in vitro. This study's findings suggest using $J$. tanjorensis as an herbal remedy to reduce the adverse effects of wound infection.

\section{ACKNOWLEDGEMENTS}

The authors appreciate the technical assistance provided by the staff of the wound care unit of the Ekiti State University Teaching Hospital (EKSUTH), Ado-Ekiti, Nigeria, during the collection of wound swabs from inpatients.

\section{REFERENCES}

Ashish KN, Kunjabihari S, Sabuja S. 2016. Comparative studies on the phytochemistry, antimicrobial and antioxidant activities of Jatropha species ( $J$. curcas L. and $J$. gossypifolia L.) of Odisha. Intl J Pharmacog Phytochem Res 8 (10): 1614-1624.

Atata RF, Sani A, Ajewole SM. 2003. Effects of stem bark extracts of Enantia chloranta on some clinical isolates. Biochem 15 (2): 84-92.

Bayode MT, Olalemi, AO, Oladejo BO. 2021. Multiple antibiotic resistant index and detection of qnrS and qnrB genes in bacterial consortium of urine samples from clinical settings. Eur J Biol Res 11 (1): 45-56. DOI: 10.5281 /zenodo.4304311.

Beni S, Amran L, Firdaus MM. 2014. Antibacterial activity of leaves extracts of Jatropha curcas, Linn against Enterobacter aerogenes. Intl J Sci Technol Res 3 (1): 129-131.

Bodunrinde RE, Oladunmoye MK, Bayode MT, Adetunji CO, Gabriel PO. 2020. Synergistic antibacterial activity of Hibiscus sabdariffa, honey and ciprofloxacin on selected uropathogens. Arch Sci Technol 1: $75-87$.

Colombini MP, Andreotti A, Bonaduce I, Modugno F. 2010. Analytical strategies for characterizing organic paint media, using gas chromatography/mass spectrometry. Acc Chem Res 43 (6): 715-727. DOI: 10.1021/ar900185f.

Clinical Laboratory Standard Institute (CLSI). 2014. Performance Standards for Antimicrobial Susceptibility Tests. Document M100517. CLSI, Wayne, PA.

Don JB, James TS, Noel RK. 2006. Bergey's manual of systematic bacteriology (Volume 2, Parts Aâ. "C, 2nd Edition). FEMS Immunol Med Microbiol 46 (3): 400-476. DOI: 10.1111/j.1574695X.2005.00055.x.

Douye VZ, Elijah IO, Medubari BN. 2013. Antibacterial activity of ethanol, crude and water extract of Chromolaena odorata leaves on $S$. typhi and E. coli. Greener J Microbiol Antimicrob 1 (2): 16-19. DOI: 10.15580/gjma.2013.2.110713950.

Ebana RUB, Andy IE, Edet UO, Anosike IK, Mbim EN, Benjamin AU. 2019. Nutritional studies and antimicrobial activities of Jatropha tanjorensis leaves extracts against Escherichia coli isolates. Intl J Inno Sci Res Technol 4 (8): 945-956

Escandon J, Vivas AC, Tang J, Rowland KJ, Kirsner RS. 2011. High mortality in patients with chronic wounds. Wound Rep Reg 19: 526528. DOI: $10.1111 / \mathrm{j} .1524-475 x .2011 .00699$.x.

Farrel KT. 1990. Spices, Condiments and Seasonings. Springer, US. DOI: 10.1007/978-1-4615-7898-7.

Gautam MK, Murthy S, Goel S, Purohit V, Sharma H, Goel RK. 2013. Evaluation of in vivo wound healing activity of Bacopa monniera on different wound model in rats. Biomed Res Intl 2013: 1-10. DOI: $10.1155 / 2013 / 972028$.

Guo S, Di-Pietro LA. 2010. Factors affecting wound healing. J Dental Res 89 (3): 219-229. DOI: 10.1177/0022034509359125.

Harbone NV. 1994. Phytochemical Methods. A Guide to Modern Techniques of Plant Analysis, 2nd ed. Chapman and Hall Ltd., London.

Hartwell JL. 1969. Plants used against cancer: A survey. Lloydia 32: 153205.

Houghton PJ, Hylands PJ, Mensah AY, Hensel A, Deters AM. 2005. In vitro tests and ethnopharmacological investigations: Wound healing as an example. J Ethnopharmacol 100: 100-107. DOI: 10.1016/j.jep.2005.07.001.

Ibekwe VI, Nnanyere NF, Akujobi CO. 2001. Studies on antibacterial activity and phytochemical qualities of extracts of orange peels. Intl $\mathrm{J}$ Environ Health Human Dev 2 (1): 41-46.

Iwalewa EO, Agbani EO. 2005. Pro-antioxidant effects and cytoprotective potentials of nine edible vegetables in Southwest Nigeria. J Med Food 8: 539-544. DOI: 10.1089/jmf.2005.8.539.

Levine NS, Lindberg RB, Mason AD, Pruitt BA. 1976. The quantitative swab culture and smear: A quick, simple method for determining the number of viable aerobic bacteria on open wounds. J Trauma 16: 8994. DOI: 10.1097/00005373-197602000-00002.

Meenakshi K, Premanadham N, Munilakshmi P, Sreenivasulu PR. 2015. Bacterial profile of post operative wound infection and antibiotic sensitivity pattern. Intl J Healthcare Sci 2 (2): 251-248. DOI: 10.5455/2320-6012.ijrms20150332.

Mohammed A, Seid ME, Gebrecherkos T, Tiruneh M, Moges F. 2017. Bacterial isolates and their antimicrobial susceptibility patterns of wound infections among in-patients and outpatients attending the 
University of Gondar Referral Hospital, Northwest Ethiopia. Intl J Microbiol 10: 1-10. DOI: 10.1155/2017/8953829.

Morales-Cabrera M, Hernandez-Morales J, Leyva-Ruelas G, SalinasMoreno Y, Soto-Rojas L, Castro- Rosas J. 2013. Influence of variety and extraction solvent on antibacterial activity of roselle (Hisbicus sabdariffa L.) calyses. J Med Plants Res 7 (31): 2319-2322. DOI: $10.5897 / \mathrm{jmpr} 12.1242$.

Mulu W, Gidey B, Chernet A, Alem G, Abera B. 2013. Hepatotoxicity and associated risk factors in hiv-infected patients receiving antiretroviral therapy at Felege Hiwot Referral Hospital, Bahirdar, Ethiopia. Ethiop J Health Sci 23 (3): 15-22 DOI 10.4314/ejhs.v23i3.4.

Murti K, Lambole V, Panchal M. 2011. Effect of Ficus hispida leaf on normal and dexamethasone suppressed wound healing, Brazil J Pharm Sci 47 (4): $855-860$. DOI: $10.1590 / \mathrm{s} 1984-$ 82502011000400023 .

National Institute of Standards and Technology (NIST). 1998 https://icsd.nist.gov/, National Institute of Standards and Technology Mass Spectral database, United States.

Ogba OM, Olorode OA, Adie GP. 2014. Bacterial pathogens associated with wound infections in Calabar, Nigeria. J Med 13 (1): 26-33. DOI: 10.18035/emj.v2i3.201.

Omoregie ES, Osagie AU. 2007. Phytochemical screening and antianemic effect of Jatropha tanjorensis leaf in protein malnourished rats. Plants Arch 7: 509-516. DOI: 10.1111/j.1745 4514.2010.00392.x.

Paul CO, Omoaruemike EO, Sikiru OO. 2013. Phytochemical screening and effect of Musa paradisiaca stem extrude on rat haematological parameters. Curr Res J Biol Sci 5 (1): 26-29. DOI: $10.19026 /$ crjbs. 5.5468
Shreshta RKC, Sharma A. 2013. Bacteriological study of wound infection and antibiotic susceptibility pattern of the isolates. Nepal J Sci Technol 14 (2): 143-150. DOI: 10.3126/njst.v14i2.10428.

Sule IO, Agbabiaka TO. 2008. Antibacterial effect of some plant extracts on selected Enterobacteriaceae. Ethnobot Leafl 12: 1035-1042.

Swarbrick T. 1997. Weeds of the Pacific Islands, Technical Paper No 209 South Pacific Commission Noumea, New Caledonia.

Tatah AJF, Ngunde PJ, Evelyn MS, Gerard N, Ndip RN. 2014. Risk factors for wound infection in health care facilities in Buea, Cameroon: Aerobic bacterial pathogens and antibiogram of isolates. Pan Afr Med J 18: 1-14. DOI: 10.11604/pamj.2014.18.6.2304.

Thomsen TR, Aasholm MS, Rudkj-bing VB, Saunders AM, Bjarnsholt T, Givskov M, Kirketerp-Muller K, Nielsen PH. 2010. The bacteriology of chronic venous leg ulcer examined by culture-independent molecular methods. Wound Rep Reg 18: 38-49. DOI: 10.1111/j.1524475x.2009.00561.x

Van Beek TA. 1999. Modern methods of secondary product isolation and analysis. In: Walton NJ, Brown DE (eds.). Chemicals from Plants, Perspectives on Plant Secondary Products. Imperial College Press, London. DOI: 10.1142/9789812817273_0003.

Vis wanathan MBG, Ananthi JDJ, Kumar PS. 2012. Antimicrobial activity of bioactive compounds and leaf extracts in Jatropha tanjorensis. Fitoterapia 83: 1153-1159. DOI: 10.1016/j.fitote. 2012.07.007.

Wong VW, Martindale RG, Longaker MT, Gurtner GC. 2013. From germ theory to germ therapy: Skin microbiota, chronic wounds, and probiotics. Plastic Reconstruct Surg 132 (5): 854-861. DOI: 10.1097/PRS.0b013e3182a3c11e.

Yamac M, Bilgili F. 2006. Antimicrobial activities of fruit bodies and/or mycelial cultures of some mushroom isolates. Pharm Biol 44 (9): 660-667. DOI: $10.1080 / 13880200601006897$. 